\title{
Nanoparticle Conjugates of Selenium Compounds: Preparation, Characterisation and Electron Transfer
}

\author{
K. I. Priyadarsini 1, ${ }^{*}$, B.G. Singh ${ }^{2}$, P.P.Phadnis ${ }^{3}$, K.C. Barick ${ }^{3}$ and P. A. Hassan ${ }^{3}$ \\ 1 UM-DAE Centre for Excellence in Basic Sciences, University of Mumbai, Kalina Campus, Santacruz (E), \\ Mumbai-400098, India \\ 2 Radiation \& Photochemistry Division; beenam@barc.gov.in \\ 3 Chemistry Division, Bhabha Atomic Research Centre, Trombay, Mumbai - 400085, India; \\ phadnisp@barc.gov.in (P.P.P.); kcbarick@barc.gov.in (K.C.B.); hassan@barc.gov.in (P.A.H.) \\ * Correspondence: k.indira@cbs.ac.in \\ + Presented at the $1^{\text {st }}$ International Electronic Conference on Catalysis Sciences, 10-30 November 2020; \\ Available online: https://eccs2020.sciforum.net.
}

Published: 10 November 2020

\begin{abstract}
One of the important features of influencing the biological applications of organoselenium compounds is its redox state, which in turn is affected by its interactions with nearby heteroatoms. To modulate the biological action of selenium in such compounds, researchers have designed new structural motifs and also developed new formulations using inorganic nanoparticles. Metal nanoparticles like gold nanoparticles (GNP) and magnetic nanoparticles (MNP) like iron oxide $\left(\mathrm{Fe}_{3} \mathrm{O}_{4}\right)$ have been extensively studied for conjugation with many heteroatoms (sulphur, nitrogen, oxygen) containing ligands. Selenium being more polarisable than sulphur can induce significant surface passivation thereby providing easy modulations in physico-chemical properties. With this aim, we investigated the physico-chemical properties of a few selenium compounds conjugated to GNP and MNP. The GNP conjugates were characterised by spectroscopic and microscopic tools like optical absorption, Raman spectroscopy, DLS, Zeta potential and TEM. The results confirmed that the selenium atom was covalently conjugated to GNP and this conjugation has not only increased their electron transfer ability but also their antioxidant ability. In another study asymmetric phenyl selenides were conjugated with MNP and characterised by XRD, TEM, DLS and zeta-potential. The radical scavenging ability of the selenium compounds improved on conjugation with the MNPs. Thus, the above studies confirmed that the redox activities of selenium compounds can be modulated on conjugating with inorganic nanoparticles like GNP and MNP, which in turn provide new avenues for delivering organoselenium compounds.
\end{abstract}

Keywords: selenium; gold nanoparticle; magnetic nanoparticle; nano-conjugates; redox reaction

\section{Introduction}

Selenium compounds find many applications in biology, medicine and materials development. As amicronutrient, it is present in humans in the form of selenoproteins like glutathione peroxidase and thioredoxinreductaseenzymes, that help in preventing cellular damage from reactive oxygen species (ROS) and regulating the redox balance in the body [1-3]. Selenium has very narrow toxicity profile for humans,from being essential to toxic in microgram concentration region. In the essential region it is being explored for many medical applications like treatment of cancer and many other endemic diseases. To explore this activity of selenium researchers are exploring new methods to enhance its redox activity by conjugating to nanoparticle systems [2,3]. Among these gold nanoparticles (GNP) and magnetic nanoparticles (MNP) are finding excessive use due to their versatile properties [4-6]. GNPs show antimicrobial activity and have been studied to develop a potential drug and gene delivery system in cancer therapy. Additionally, they show photothermal 
effects, which provide additional advantage. MNPs, in particular $\mathrm{Fe}_{3} \mathrm{O}_{4}$, are widely used as a carrier for delivery of drugs and biomolecules due to their unique magnetic properties, low toxicity, and biodegradability $[5,6]$. Both the particles have reactive surface that can be easily functionalized with biocompatible molecules and pharmaceutical agents. Several ligands containing heteroatoms like nitrogen, sulfur, phosphorous etc have been extensively employed for surface passivation of inorganic materials. In particular, molecules with thiol functional groups have shown excellent ability to bind with gold surfaces via chemisorption. Selenium is softer and more polarizable than sulfur [2], therefore it is expected to interact more strongly. Selenium compounds attached to GNPs have been found to exhibit better anti-cancer activity than unconjugated selenium compounds [7-9]. GNPs has also been found to be very efficient substrate for detectionof selenium even in nanomolarrange, using surface enhanced Raman scattering(SERS) [7]. Recently Gold-Se conjugates are being developed asefficientnanoprobes for detection of signaling associated with cancer progression [8]. Thus preparing nanoconjugates with selenium compounds is emerging as an alternative and efficient approach to modify their physico-chemical and biochemical properties. With this aim, a few selenium compounds have been conjugated with GNPs and MNPs and evaluated for radical scavenging activity. For GNP-Selenium studies, we have employed bis-2-ethanolselenide (EOH-Se-EOH) as a model SE compound and for MNP-Selenium studies we used various Se containing aminoacid derivatives such as selenoglycine derivative $(\mathrm{SeG})$ and selenoserine derivative (SeS). Synthesis of selenium compounds are reported in our earlier papers $[10,11]$. The chemical structure of selenium compound used for GNP interaction is given in scheme 1.

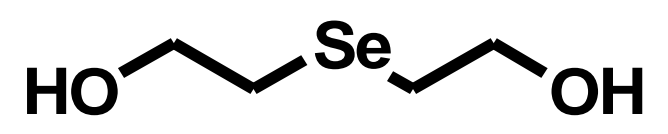

Scheme 1. Chemical structure of EOH-Se-EOH.

\section{GNP-Selenium Studies}

To understand the interaction of selenium compounds, first GNPs were prepared by reducing potassiumtetrachloroaurate(III), $\mathrm{K}\left[\mathrm{AuCl}_{4}\right]$ with trisodium citrate in nanopure water under constant stirring and controlled heating.A deep wine red colored solution was obtained after formation of GNPS which show characteristic optical absorption with a $\lambda_{\max }$ of $522-524 \mathrm{~nm}$, due to the localised surface plasmon resonance. The average hydrodynamicdiameter of the GNPs, as obtained from dynamic light scattering (DLS)was found to be of $15 \pm 3 \mathrm{~nm}$ and the zeta-potential of the particles was determined to be $-39.5 \pm 2.9 \mathrm{mV}$. These GNPs were treated with the symmetric selenoether, bis2-ethanolselenide (EOH-Se-EOH). This leads to a ligand exchange reaction at the surface of GNPs and a consequent shift in the absorption maximum to $650-680 \mathrm{~nm}$ (blue colored). The changes in the optical properties of GNP upon binding with selenoether have been used to obtain the binding constant of the ligand [12]. Following the absorption changes at $520 \mathrm{~nm}$, as a function of EOH-Se$\mathrm{EOH}$ concentration and fitting these datato a linear plot as per Benesi-Hildebrand equation yielded a binding constant of $5.9 \pm 0.2 \times 10^{2} \mathrm{M}^{-1}$. Upon binding with selenother, the hydrodynamic size and zeta potential of the GNP changed to $26 \pm 2 \mathrm{~nm}$ and $-27.6 \pm 0.8 \mathrm{mV}$ respectively.

SERS studies of GNP and GNP conjugated EOH-Se-EOH showed very interesting features. The C-Se-C stretching vibration of the compound at $551 \mathrm{~cm}^{-1}$ showed a shift of $9.7 \mathrm{~cm}^{-1}$ upon binding to GNP. The $\mathrm{CH}_{2}$ bending vibration, which showed multiple peaks from 1015 to $1558 \mathrm{~cm}^{-1}$, also showed small shifts on binding to GNP, indicating that both the selenium as well as the alkyl chain of EOHSe-EOH interactwith GNP. This would affect the electron density and redox potential of the selenium. To assess this, electron transfer reaction between EOH-Se-EOH with ABTS.+ radical were studied in the presence and absence of GNP and was monitored by spectrophotometry [12]. ABTS.+ has a strong absorption band from 400 to $800 \mathrm{~nm}$ and is a very long lived radical. It can be prepared by the reaction of $\mathrm{ABTS}^{2+}$ with an oxidant, like $\mathrm{K}_{2} \mathrm{~S}_{2} \mathrm{O}$. The absorption-time plot as observed at $820 \mathrm{~nm}$, did not show significant decay within the measured time scale, in the absence of any additive and the estimated decay constant was $3.7 \times 10^{-6} \mathrm{~s}^{-1}$. In presence of GNP, the decay constant increased to $1.1 \times 10^{-4} \mathrm{~s}^{-1}$ and 
in presence of EOH-Se-EOH the decay constant was $8 \times 10^{-5} \mathrm{~s}^{-1}$. However, in the presence of GNPEOH-Se-EOH conjugate, the decay showed a significant increase to $2.1 \times 10^{-3} \mathrm{~s}^{-1}$. This clearly confirmed that GNP conjugation increased the electron transfer ability of the selenium compound. The increase in the electron transfer ability on conjugation to GNP has been attributed to increase in the stability of the selenium centred radical formed on the GNP surface in a separate study involving pulse radiolysis [12]. A schematic illustration of the binding of EOH-Se-EOH on gold nanoparticle surface and consequent changes in the color of the nanoparticle suspension is given in Figure 1.

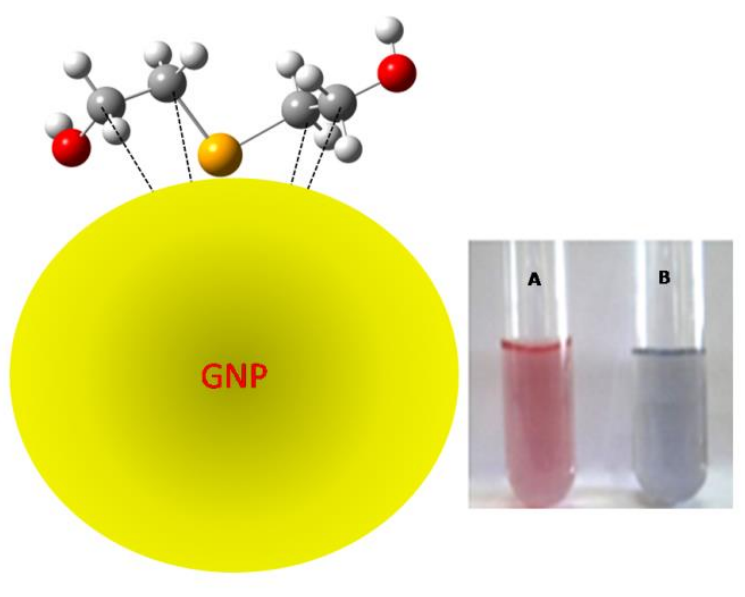

Figure 1. Schematic representation of GNP bound EOH-Se-EOH and photographs of the GNP suspension before (A) and after (B) conjugation with selenium compound.

\section{MNP-Selenium Studies}

In the second study, $\mathrm{Fe}_{3} \mathrm{O}_{4}$ magnetic nanoparticles (MNPs) coated with glycine were employed to conjugate selenium compounds. Glycine coating not only provides stabilization to MNPs but also leaves freely exposed amine groups, improving water dispersability. These MNPs (TEM 10 nm), were prepared byco-precipitation method as reported earlier [13] and separated from the supernatant using a permanent magnet. Since magnetic nanoparticles can be separated using a permanent magnet, this offers an attractive way to purify the suspension from any unbound molecules or reaction by-products. These MNPs were characterized by IR spectroscopy which showed the characteristic vibrations at $588 \mathrm{~cm}^{-1}(\mathrm{Fe}-\mathrm{O}), 1400 \mathrm{~cm}^{-1}\left(\mathrm{U}_{\mathrm{sym}} \mathrm{COO}^{-}\right)$and $1586 \mathrm{~cm}^{-1}\left(\mathrm{Uasym}_{\mathrm{COO}}\right)$. The morphology of the particles were identified from TEM (roughly spherical particle of average size $\sim 10$ $\mathrm{nm}$ ), and the magnetisation studies indicate superparamagnetic behaviorwith a maximum magnetization of $65.2 \mathrm{emu} / \mathrm{g}$ at $300 \mathrm{~K}$. The aminoacid passivated MNPs were further conjugated with selenium containing $\mathrm{N}$-acetyl alpha aminoacid compounds, $\mathrm{PhSeCH}_{2} \mathrm{CONHCH}_{2} \mathrm{COOH}(\mathrm{SeG})$ and $\mathrm{PhSeCH} 2 \mathrm{CONHCH}\left(\mathrm{CH}_{2} \mathrm{OH}\right) \mathrm{COOH}(\mathrm{SeS})$ which were synthesized as reported elsewhere [11]. The conjugation of the selenium compounds to the MNPs were carried by well-known bio-conjugation reaction EDC-NHS coupling between the carboxyl group of Se compound and amine functional group on MNP surface [13]. Figure 2 shows a schematic illustration of the conjugation of selenium containing N-acetyl alpha aminoacids on glycine passivated MNPs. 

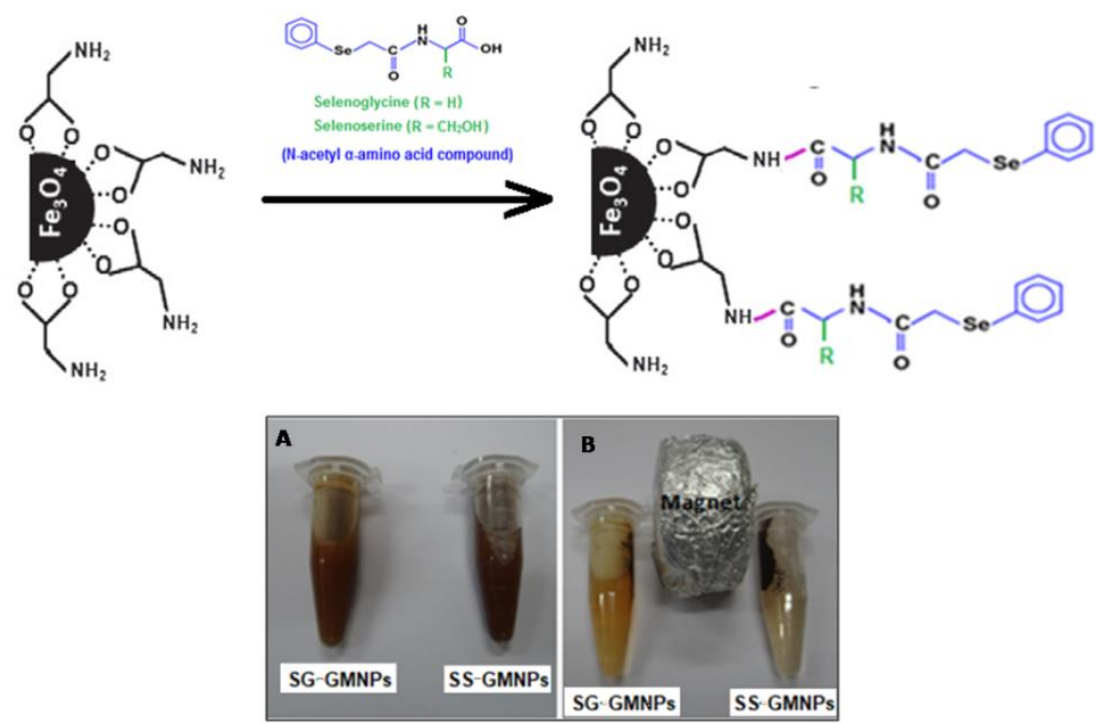

Figure 2. Schematic representation of conjugation of $\mathrm{N}$-acetyl $\alpha$-amino acid compounds on GMNPs.Photographs of aqueous suspension of SG-GMNPs and SS-GMNPs in the absence (A) and resence (B) of magnetic field (filed strength of table top magnet: $0.25 \mathrm{kOe}$ ). The agglomeration of the particles in the presence of a magnet can be clearly seen in the photograph.

Conjugation of SeG and SeS onto the surface of MNPs did not affect the crystal structure of $\mathrm{Fe}_{3} \mathrm{O}_{4}$ as assessed by XRD. From ICP-AES analysis, it was found that about 80 and $70 \mu \mathrm{g} / \mathrm{g}$ of SeG and SeS respectively could be loaded onto MNPs. DLS measurements indicated that the average hydrodynamic diameter of the particles was 104 and $115 \mathrm{~nm}$ and the zeta-potential of -20 and $-25 \mathrm{mV}$ at pH 7.4 for SeG-MNPs and SeS-MNPs, respectively. Further, both SeG-MNPs and SeS-MNPs showedgood magnetic field responsivity under external magnet.

The redox behavior of the MNP loaded selenium compounds were evaluated by DPPH radical scavenging assay. DPPH radical is a stable radical and gets reduced by compounds that have ability to donate an electron or hydrogen atom. The reaction is followed by monitoring the absorbance at $517 \mathrm{~nm}$ due to DPPH radical, which becomes pale yellow on reduction. When SeG-MNP and SeSMNP were evaluated for DPPH scavenging assay, the results indicated that the selenium compoundsnot only retained their original DPPH scavenging activity but also improved marginally on binding to MNP.

\section{Conclusions}

In conclusion, we have demonstrated the ability of various inorganic nanocarriers for binding of organoselenium compounds and its impact on electron transfer behavior. In particular, the above studies indicate that selenium compounds can be easily conjugated to different types of nanoparticles such as GNPs or MNPs. Such binding with nanoparticles like GNP significantly improves their electron transfer ability. This can therefore provide novel way of improving their antioxidant activity. Similarly, organoselenium compounds can also be conjugated to $\mathrm{Fe}_{3} \mathrm{O}_{4} \mathrm{MNPs}$. Such conjugation although did not significantly increase their electron transfer ability, glycine conjugation gives water solubility and their magnetic nature provide targets specificity. Overall these studies provide a new avenues to modulate the biological properties of selenium compounds, using inorganic nanocarriers.

Author Contributions: “Conceptualization, K.I.P. and P.A.H; methodology, B.G.S., P.P.P. and K.C.B.; data curation, B.G.S., P.P.P. and K.C.B.; writing-review and editing, K.I.P. and P.A.H.; supervision, K.I.P. All authors have read and agreed to the published version of the manuscript." 
Acknowledgments: The authors thank the Director, UM-DAE-CEBS, the Head, RPC Division BARC, and the Associate Director, Chemistry Group, BARC, for their support and encouragement. KIP thanks DAE for the Raja Ramanna Fellowship.

Conflicts of Interest: The authors declare no conflict of interest.

\section{References}

1. Papp, L.V.; Lu, J.; Holmgren, A.; Khanna, K.K.From selenium to selenoproteins: synthesis, identity, and their role in human health. Antioxid. Redox. Signal. 2007, 9, 775-806.

2. Jain, V.K.; Wessjohan, L.A.; Santi, C.; Singh, H.B.; Wirth, T.; Lippolis, V.; Holmgren, A. Organoselenium Compounds in Biology and Medicine; RSC: London, UK, 2018.

3. Plano, D.; Baquedano, Y.; Ibáñez, E.; Jiménez, I.; Palop, J.A.; Spallholz, J.E.; Sanmartín, C. Antioxidant-prooxidant properties of a new organoselenium compound library. Molecules 2010, 15, 7292-7312.

4. Dykman, L.A.; Khlebtsov, N.G.Gold nanoparticles in biology and medicine: recent advances and prospects.Acta Naturae. 2011, 3, 34-55.

5. Laurent, S.; Forge, D.; Port, M.; Roch, A.; Robic, C.; Elstand, L.V.R.; Muller, N. Magnetic iron oxide nanoparticles: Synthesis, stabilization, vectorization, physicochemical characterizations, and biological applications.Chem. Rev.2008, 108, 2064.

6. Barick, K.C.; Hassan, P.A. Glycine passivated $\mathrm{Fe}_{3} \mathrm{O}_{4}$ nanoparticles for thermal therapy,J. Coll. Interface Sci.2012, 369, 96.

7. Cao, G.; Xu, F.; Wang, S.; Xu, K.; Hou, X.; Wu, P. Gold Nanoparticle-Based Colorimetric Assay for Selenium Detection via Hydride Generation.Anal. Chem.2017, 89, 4695-470.

8. Li, T.;Li, F.;Xiang, W.;Yi, Y.;Chen, Y.;Cheng, L.;Liu, Z.;Xu, H. Selenium-containing Amphiphiles Reduced and Stabilized Gold Nanoparticles: Kill Cancer Cells via Reactive Oxygen Species.ACS Appl. Mater. Interfaces 2016,8, 22106-22112.

9. Luan, M.;Shi,M.;Pan,W.;Li, N.;Tang,B. A gold-selenium-bonded nanoprobe for real-time in situ imaging of the upstream and downstream relationship between uPA and MMP-9 in cancer cells.Chem. Commun.2019, 55, 5817-5820.

10. Kumar, P.V.;Singh, B.G.;Phadnis, P.P.;Jain,V.K.;Priyadarsini, K.I. Effect of Molecular Interactions on Electron-Transfer and Antioxidant Activity of Bis (alkanol) selenides: A Radiation Chemical Study. Chem. A Eur. J. 2016, 22, 12189-12198.

11. Prabhu, C.P.; Phadnis, P.P.; Wadawale, A.;Priyadarsini, K.I.jJain, V.K. One-pot synthesis of phenylseleno $\mathrm{N}$-acetyl $\alpha$-amino acids: Supra-molecular self-assembling in organoselenium compounds. J. Organomet. Chem.2013, 140, 745-746/

12. Kumar, P.V.;Singh, B.G.;Ballal, A.;Jain,V.K.; Iwaoka, M.;Priyadarsini, K.I.Gold nanoparticles (GNP) induced redox modulation in organoselenium compounds: distinction between cyclic vs. linear structures.RSC Adv.2016, 6, 69501-508.

13. Barick, K.C.;Dutta, B.; S. Gawali, L.;Phadnis, P.P.;Priyadarsini, K.I.;Jain, V.K.;Hassan,P.A.Phenylseleno Nacetyl $\alpha$-amino acids conjugated magnetic nanoparticles: Synthesis, characterization and radical scavenging ability.Chem. Lett. 2020, 49, X.

Publisher's Note: MDPI stays neutral with regard to jurisdictional claims in published maps and institutional affiliations.

(C) 2020 by the authors. Submitted for possible open access publication under the terms and conditions of the Creative Commons Attribution (CC BY) license (http://creativecommons.org/licenses/by/4.0/). 